\title{
MIXING BETWEEN THE CORE AND THE ENVELOPE IN STARS WITH DEEP CONVECTION ZONES*
}

\author{
DAIICHIRO SUGIMOTO \\ Institute of Earth Science and Astronomy, College of General Education, \\ University of Tokyo, Komaba, Meguro, Tokyo, Japan \\ and \\ KEN-ICHI NOMOTO \\ Dept. of Astronomy, University of Tokyo, Tokyo, Japan
}

\begin{abstract}
The extensive surface convection zone of a red giant star becomes deeper and deeper, as the star evolves. In some cases it reaches the bottom of the hydrogen-rich envelope and even penetrates into the stellar core. From the standpoint of entropy distribution in the star, the structure of the convective envelope and the mechanism of its penetration into the core are summarized. Possibilities of the penetration are discussed for stars of masses in the range of 1-60 $M_{\odot}$ over their entire lifetimes of evolution. Effects of uncertainty in the mixing length theory of convection, neutrino loss and thermal instability of helium-burning shell are studied also. When the convection penetrates into the core, material of the core is brought up to the stellar surface and the star will be a peculiar star. It is concluded that there are possibilities to interpret the origin of peculiar stars with luminosities fainter than $7 \times 10^{4} L_{\odot}$, but that the more luminous ones do not originate from the penetration of surface convection.
\end{abstract}

\section{Introduction}

Development of an extensive surface convection zone was found by Hoyle and Schwarzschild (1955) in their study of evolution of less massive stars through a giant branch of a globular cluster. They discussed that an envelope in radiative equilibrium cannot be consistent with a photospheric boundary condition in the case of red giant stars, and that it requires the development of the extensive surface convection zone.

This situation is understood more intuitively, though more roughly, as follows. We approximate the photospheric boundary condition by $\kappa_{\mathrm{ph}} \varrho_{\mathrm{ph}} R \simeq \frac{2}{3}$, where $\kappa, \varrho$ and $R$ denote opacity, density and the stellar radius, respectively, and the subscript 'ph' refers to the photospheric value. A condition that the mean density of the star is higher than the photospheric density is expressed as

$$
M>(4 / 3) \pi R^{3} \varrho_{\mathrm{ph}} \simeq(8 / 9) \pi R^{2} \kappa_{\mathrm{ph}}^{-1},
$$

where $M$ denotes the mass of the star. This condition implies that there should be an upper limit to the stellar radius. It corresponds to the Hayashi limit in the HR diagram (Hayashi and Hōshi, 1961), when computed quantitatively.

Since the polytropic index in a convective region is always smaller than that in a radiative region, an envelope with a surface convection zone has a relatively small

* This paper was presented by Daiichiro Sugimoto. 
radius (Chandrasekhar, 1939; Hayashi et al., 1962). Thus, the emergence of the convection zone is consistent with the limitation to the stellar radius.

In the present paper, we shall use the notions of the core and the envelope, dividing a star at the hydrogen-burning shell. As the star evolves, the central condensation of matter becomes stronger in the core, because the temperature and/or the electron degeneracy in the central region must increase. However, the hydrogen-burning shell hardly contracts, because its temperature must not be higher than the hydrogenburning temperature $\left(2-10 \times 10^{7} \mathrm{~K}\right)$. Since the volume of the core does not change much, the density and thus the pressure near the hydrogen-burning shell become lower, while the central condensation proceeds in the core. In order to sustain the envelope against gravity, the bulk of the envelope should be brought to a region of weak gravity, i.e., of a large radial distance from the center.

Since the radius of the star is bounded as discussed above, the convection in the envelope becomes deeper and deeper in order to push the bulk of the envelope to the relatively outer portion of the envelope. It is our problem whether the convection in the envelope grows deep enough to penetrate into the core, and whether the convective mixing brings material of the core to the stellar surface.

In the next two sections, the mechanism of penetration of convection and nature of the convective envelopes will be summarized. In the later sections, existing stellar models will be discussed and compared one another. Some of new models will be incorporated also, which we computed recently and details of which are in preparation for publication. In the final section, concluding remarks will be given concerning the possible origin of peculiar stars.

\section{Mechanism of the Penetration of Convection}

It is convenient to discuss the convection zone in terms of entropy distribution through the star. In a chemically homogeneous region in radiative equilibrium, the entropy is increasing outward, while it stays constant in adiabatic convection and even decreases outward in superadiabatic convection. We shall approximate that the chemical composition and the energy flux $L_{r}$ change discontinuously at the interface between the core and the envelope, where a hydrogen-burning shell lies (active or inactive). Quantities at the interface will be denoted by the subscript 1, adding $e$ or $i$ to indicate the external or internal side, if necessary. The distribution of entropy is illustrated in Figure 1. We shall use different definitions of entropy for the core and the envelope. Entropy defined for the core will be denoted by $s^{\text {(core) }}$ and one defined for the envelope by $s$. Non-dimensional value of the entropies will be denoted by $\sigma \equiv(H / k) s$, where $H$ and $k$ denote the mass of hydrogen and the Boltzmann constant. Anyhow, we shall assume that there exists one-to-one correspondence between $s_{1 i}^{\text {(core) }}$ and $s_{1 e}$, or $\sigma_{1 i}^{\text {(core) }}$ and $\sigma_{1 e}$ under continuity of pressure and temperature across the point 1. Practically, $s$ is defined with the quantum statistical zero-point, so that it is continuous even in the ionization and dissociation zones. We shall use the definition of $s^{\text {(core) }}$ as given in Sugimoto (1970a). 


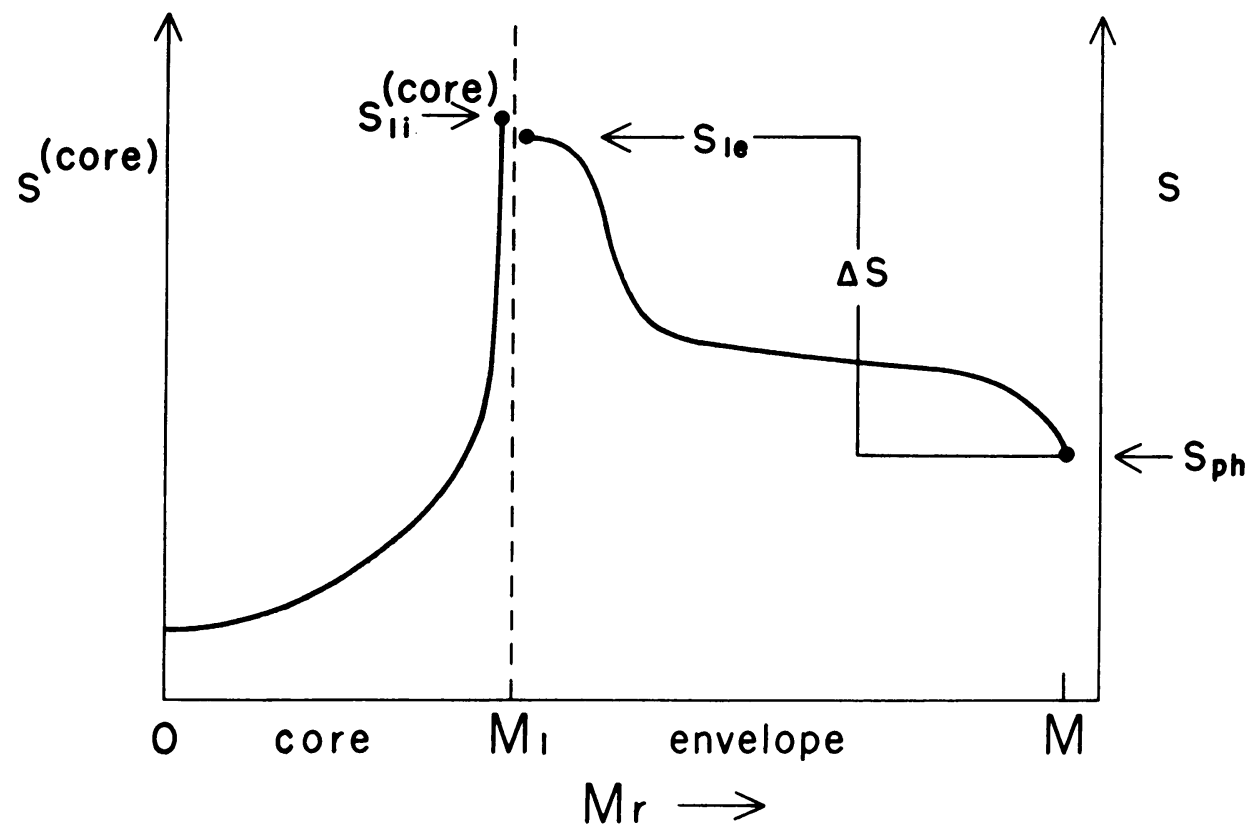

Fig. 1. Illustration of entropy distribution in the star.

From the boundary conditions at the photosphere $\kappa_{\mathrm{ph}} \varrho_{\mathrm{ph}} R \simeq 2 / 3$ and $L=\pi a c R^{2} T_{\mathrm{eff}}^{4}$, where $L$ and $T_{\text {eff }}$ denote luminosity and effective temperature of the star, the entropy at the photosphere is computed. Near the photosphere of the red giant stars, $\kappa_{\mathrm{ph}}$ is so small that the radiation pressure is negligible in many cases. In such cases, the entropy at the photosphere is expressed as

$$
s_{\mathrm{ph}}=(k / \mu H) \ln \kappa_{\mathrm{ph}} R^{1 / 4} L^{3 / 8}+\text { const },
$$

where $\mu$ denotes the mean molecular weight. In the super-adiabatic envelope, entropy takes the maximum value $s_{\mathrm{ph}}+\Delta s$ at the bottom of the convective zone. The structure of the core including the stellar luminosity depends only slightly on the structure of the envelope (Paczyński, 1970a; Sugimoto, 1970a). The entropy $s_{1 e}$ determined from the $s_{1 e}^{(\text {core })}$ should satisfy the condition,

$$
s_{1 e} \leqslant s_{\mathrm{ph}}+\Delta s .
$$

If the equality holds in the condition (3), the convection reaches just the bottom of the hydrogen-rich envelope. If the condition (3) is violated, the stellar structure is inconsistent. If we assume a smaller value of the core mass $M_{1}$, we can obtain a consistent structure. This implies that the convection should have penetrated into the core. Such a treatment has been made by Stothers and Chin (1969) and by Sugimoto (1970a, b, 1971). They fitted their core model to envelope at the hydrogenburning shell.

In an actual star, the hydrogen content and $L_{r}$ change continuously across the 
hydrogen-burning shell. The stellar structure near a thin nuclear burning shell is almost stationary, if the independent variables are taken to be $M_{r} / M_{1}$ and $t$, or $r$ and $t$ (Eggleton 1967), where $M_{r}$ denotes mass contained in a sphere of radius $r$, and $t$ denotes time of evolution. Such a nature was extensively used by Paczyński (1970b) and by Uus (1970a, 1971) to compute the model envelopes, into which the thin hydrogen-and helium-burning shells were incorporated. Though their envelope is described by ordinary differential equations, the so-called gravitational energy generation is included to a good approximation. They integrated such envelopes down to a point just interior to the helium-burning shell, where the core model is fitted to the envelope. Uus $(1971,1972 \mathrm{c})$ discussed the penetration of convection into the hydrogen-burning shell in detail. We shall consider inward integration of the envelope. When we reach the region with appreciable hydrogen-burning, $L_{r}$ is decreasing inward. Then, the radiative temperature gradient is diminished and the convection stops. Moreover, the gradient of the mean molecular weight in the hydrogen-burning shell makes this shell more convectively stable. This tends to inhibit the convection from penetrating into the core.

In some cases, however $s_{1 i}^{\text {(core) }}$ and then $s_{1 \mathrm{e}}$, which are computed from the core model, become larger and larger, until the condition (3) is strongly violated (Sugimoto, 1970b). In principle, we can compute a structure for which pressure is continuous across the point 1 but the temperature jumps there. If $s_{1 i}^{\text {(core) }}$ becomes so large that $\varrho_{1 i}$ is smaller than $\varrho_{1 e}$ in spite of the difference in the mean molecular weights, the Rayleigh-Taylor instability takes place. In view of the strong dependence of $\varrho_{1 i}^{\text {(core) }}$ on the core mass (Sugimoto 1970b), the violation of the condition (3) may be considered to lead the mixing between the core and envelope, though detailed computation has never been made.

\section{Structure of Envelope}

In order to apply the condition (3), it is necessary to estimate the increase of entropy $\Delta s$ in the envelope. At present there are no other means than the mixing length theory of convection (Böhm-Vitense, 1958), for which a parameter related with the efficiency of the convective energy transport must be assumed. Usually, it is described by $\alpha_{p}=l / H_{p}$ or $\alpha_{e}=l / H_{e}$, i.e., by the ratio of the mixing length $l$ to the scale height of pressure $H_{p}$ or of density $H_{\boldsymbol{e}}$.

When $\alpha_{p}=1.0$, the superadiabaticity is so large that an inversion of density appears (Paczyński and Ziołkowski 1969, 1970a). The density inversion is not irrational by itself, because the hydrostatic equilibrium is established with this density inversion. The Rayleigh-Taylor instability takes place in this layer of density inversion, which corresponds just to the convective instability.

What happens, if we use $\alpha_{\ell}$ with the density scale-height instead of the pressure scale-height? From the physical point of view, the mixing length must be smaller than the distance to the stellar surface and/or to the center of the star. Imposing this limitation, Uus (1972a) integrated some envelopes with $\alpha_{e}=0.5$ and 1.0 , and obtained the density inversion. Moreover, the global nature such as the relations among $\Delta s$, 
luminosity and the core mass is essentially the same as those obtained for the pressure scale-height, if the value of $H_{o}$ is adjusted appropriately. Uus (1972b) has also made a non-local treatment of convection, but the global nature in the deep interior is hardly affected.

Thus, the most important uncertainty left to us is the choice of a value of $\alpha_{p}$. In many computations of stellar evolution, $\alpha_{p}$ is assumed to lie in the range of $1-2$, for which the computed giant branch can be fitted with observed HR diagram. However, such a comparison is made only for stars of relatively small luminosity as compared with the local critical luminosity,

$$
L_{\mathrm{cr}}\left(M_{r}\right)=\frac{4 \pi G c}{\varkappa} M_{r}
$$

at the bottom of the convective envelope (Nomoto and Sugimoto, 1972). In such stars $\Delta s$ is relatively small and weakly dependent on $\alpha_{p}$. Moreover, $\alpha_{p}$ may not be constant throughout the convection zone. Thus, we can not draw any definite conclusion concerning the value of $\alpha_{p}$.

Nomoto and Sugimoto (1972) studied the dependence of $\Delta s$ on the value of $\alpha_{p}$ as well as on the mass $M$, core mass $M_{1}$, and luminosity of the star. Their results are summarized as follows. The value of $\Delta s$ is larger, as the ratios of the luminosity to the local critical luminosities, $L / L_{\mathrm{cr}}(M)$ and $L / L_{\mathrm{cr}}\left(M_{1}\right)$ are larger. Where $\Delta s$ is large, its value depends strongly on the value of $\alpha_{p}$. For $\alpha_{p}=1.0$, the effect of superadiabaticity is essential. For $\alpha_{p}=1.5$, however, the envelopes are not much different from adiabatic one, unless $L / L_{\mathrm{cr}}\left(M_{1}\right)$ is close to unity. Details may be found in graphs given by Nomoto and Sugimoto (1972).

\section{Massive Stars}

In this section we shall discuss the stars of mass greater than $8 M_{\odot}$, for which electrons are non-degenerate in the carbon-oxygen core.

\subsection{Phases before the exhaustion of Carbon}

Detailed Henyey-type computation including the hydrogen-rich envelope was made by Ziołkowski (1972) for the stars of masses 15 and $30 M_{\odot}$ up to the carbon ignition, and for the star of mass $60 M_{\odot}$ up to the exhaustion of helium. The convective envelope was treated in detail with $\alpha_{p}=1.0$.

Distribution of hydrogen content in the star is illustrated in Figure 2. The intermediate zone, where the hydrogen content changes smoothly, has been produced in the core hydrogen-burning phase. Just after the helium ignition, the surface convection reaches the outer part of the intermediate zone. Then a part of helium in the intermediate zone is mixed into the convective envelope, as illustrated in Figure 2. The hydrogen concentration in the envelope, which was $X_{e}=0.700$ initially, are decreased to $0.682,0.630$ and less than 0.604 , respectively, for the stars of masses 15 , 30 and $60 M_{\odot}$. 


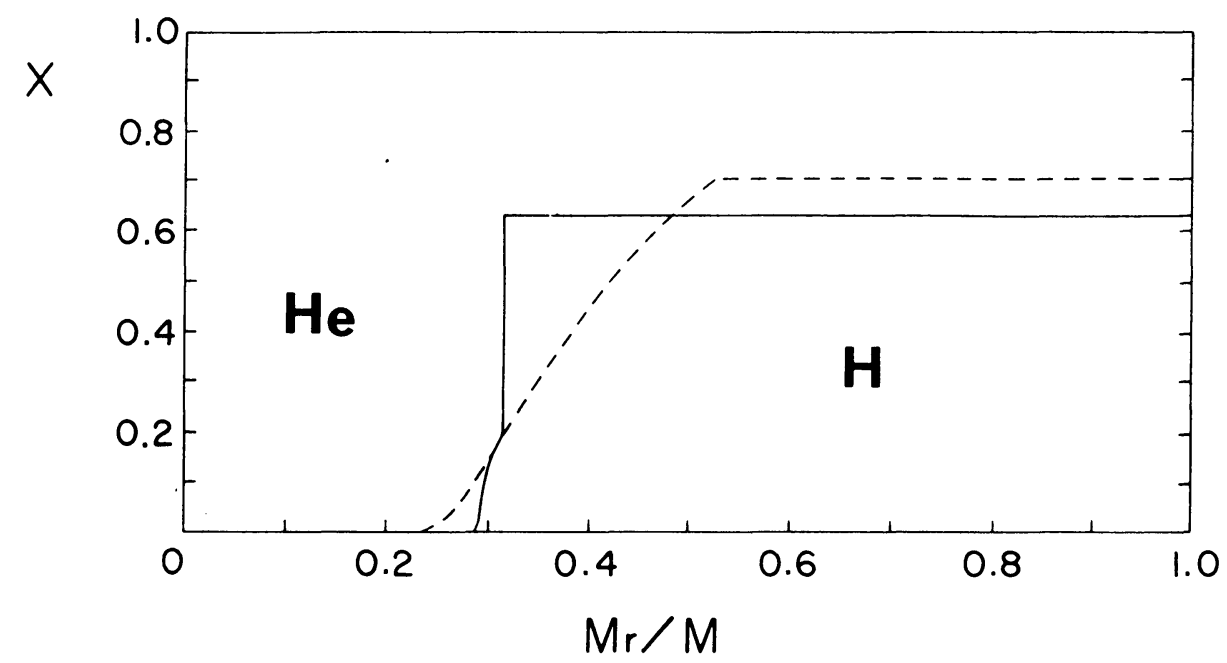

Fig. 2. Distribution of hydrogen concentration $X$ in the star changes from the dashed profile to the solid one. Computed by Ziolkowski (1972) for the star of $30 M_{\odot}$.

In the main phase of the core helium burning, the surface convection retreats. From the helium exhaustion, the star becomes a red giant again and the surface convection becomes deeper. For the stars of 15 and $30 M_{\odot}$, the convection does not reach the hydrogen-burning shell, before the ignition of carbon. For the star of $60 M_{\odot}$, the bottom of convection may reach the active hydrogen-burning shell, though Ziołkowski did not compute such stages in detail.

As shown in Figure 2, the mass $M_{r}$ at the inner edge of the intermediate zone increases as a result of the hydrogen-shell burning. Then, an almost discontinuous profile of the hydrogen distribution is realized. Stothers and Chin (1969) and Sugimoto (1970a, b) computed models, assuming such a discontinuous distribution of hydrogen. Both of them assumed adiabatic convection in the envelope. Stothers and Chin fitted such envelopes to homogeneous cores neglecting gravitational energy release. Sugimoto made Henyey-type computation of the core using the boundary conditions at the outer edge of the core,

$$
\left(\frac{G M_{r} \varrho}{r P}\right)_{1 e}=(n+1)_{1 e}= \begin{cases}4 & \text { (radiative) } \\ (n+1)_{\mathrm{ad}} & \text { (convective) }\end{cases}
$$

where $n$ and $P$ denote the polytropic index and pressure, respectively, and the subscript 'ad' denotes the adiabatic value.

In the stars of $12 M_{\odot}$ (Sugimoto, 1970b) and $15 M_{\odot}$ (Stothers and Chin, 1969), convection does not penetrate into the core. In the star of $30 M_{\odot}$ both results by Stothers and Chin, and by Sugimoto indicate that the convective envelope begins to penetrate into the core just before the ignition of carbon burning. As shown in Figure 3, helium of $1.2 M_{\odot}$ is mixed from the outer part of the core into the convective envelope in early stages of the core carbon burning (Sugimoto, 1970b). 


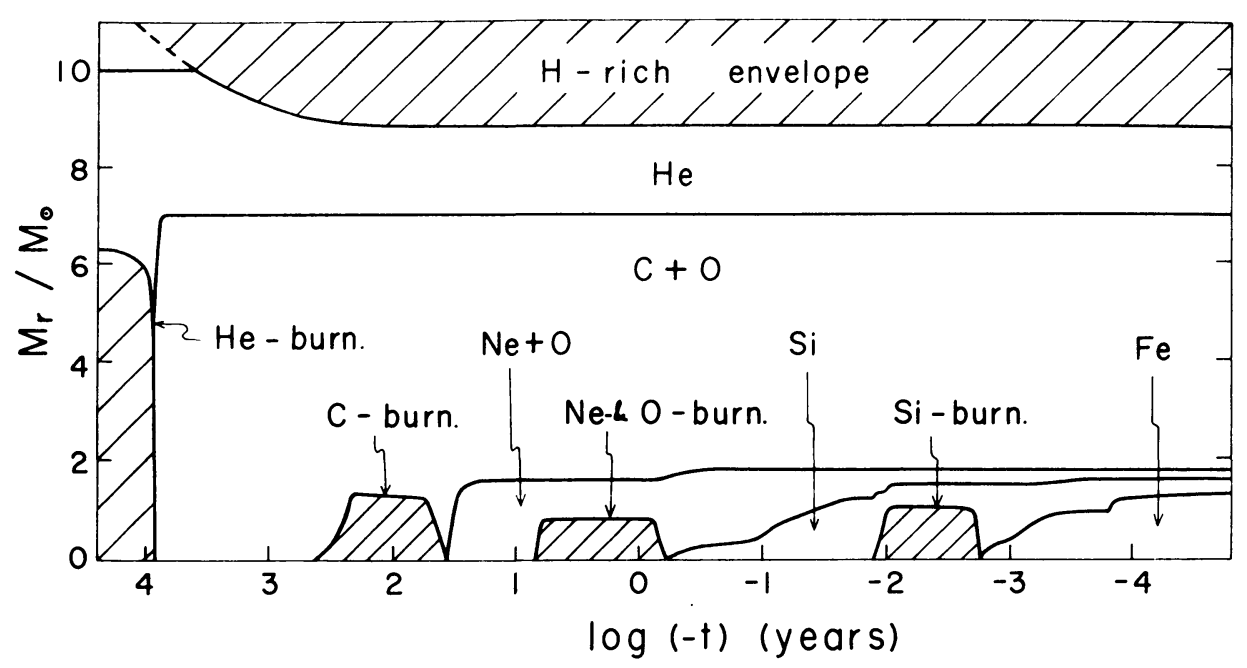

Fig. 3. Chemical evolution of the star of $30 M_{\odot}$ computed by Sugimoto 1970b) and by Sugimoto and Nomoto (1974). Neutrino loss is taken into account. Shaded regions are in convective equilibrium. A part of the hydrogen-rich envelope is omitted from the top of the figure.

Though the penetration of convection is somewhat overestimated in the treatment by Sugimoto (Nomoto and Sugimoto, 1972), it does not reach the carbon-oxygen zone by a large margin.

Nomoto (1974) computed the evolution of a $60 M_{\odot}$ star with $\alpha_{p}=1.0$, using boundary conditions which are essentially the same as Equation (5). They assumed a core mass of $30 M_{\odot}$, which is somewhat larger than the core mass of $25.05 M_{\odot}$ obtained by Ziołkowski (1972). A discontinuous change in the distribution of hydrogen was assumed. After helium has been almost exhausted in the central region, the hydrogenshell burning remains inactive, entropy at the edge of the core becomes larger and larger, and then the convective envelope penetrates into the core. About $5 M_{\odot}$ of helium and $2 M_{\odot}$ of carbon and oxygen are mixed into the envelope.

Such a star may be a peculiar star. Lifetime of this star after the mixing of carbon and oxygen is as short as $3 \times 10^{3} \mathrm{yr}$, because extensive neutrino loss accelerates the evolution in later phases. The lifetime of the core helium burning is $2.5 \times 10^{5} \mathrm{yr}$, which the star spends as a red supergiant (Ziołkowski, 1972). Then, the ratio of the peculiar stars to the red supergiants will be 0.01 .

\subsection{LATER PHASES AND THE TIMESCALE OF MIXING}

If the entropy distribution near the outer edge of the core is expressed as a function of $M_{r} / M_{1}$ and $t$, it remains almost stationary, even when $M_{1}$ is being reduced by mixing between the core and envelope. Then, the entropy of a mass element in the outer part of the core should have been greatly increased, before it is mixed into the envelope (Sugimoto, 1970a). This is accomplished by absorbing radiative heat flux from the interior, which takes a time of heat transfer $\tau_{h}\left(\Delta r=H_{p}\right)$ over unit scale- 
height of pressure. Thus, the rate of mixing is limited by

$$
-\frac{\mathrm{d} \ln M_{1}}{\mathrm{~d} t}<\frac{\Delta M / M_{1}}{\tau_{h}\left(\Delta r=H_{p}\right)},
$$

where $\Delta M$ denotes mass contained in unit scale-height of pressure. Typical values are $\Delta M / M_{1} \sim 10^{-3}$ and $\tau_{h} \sim 10 \mathrm{yr}$. near the edge of the core, and the timescale of mixing $\left|\mathrm{d} t / \mathrm{d} \ln M_{1}\right|$ amounts to $10^{4} \mathrm{yr}$.

After the carbon-burning phase, stellar evolution is greatly accelerated by copious emission of neutrinos. Typical timescale of evolution is $40 \mathrm{yr}$ for the star of mass $30 M_{\odot}$, which is too short compared with the timescale of mixing. For the star of mass about $12 M_{\odot}$, the corresponding lifetime is $1500 \mathrm{yr}$. However, the convection does not reach the bottom of the envelope even in the phase of neon burning. Anyhow, the mixing is negligible after the carbon-burning phase of massive stars (Sugimoto, 1970b; Stothers and Chin, 1969).

\subsection{EVOLUTION WITHOUT NEUTRINO LOSS}

Hayashi et al. (1962) discussed an inconsistency between the observed number of red and yellow supergiants in star clusters and their lifetimes computed with neutrino loss. Now, this inconsistency seems to be resolved by the interpretation that most members of the red supergiants are in the phase of helium burning (Stothers and Chin, 1969; Stothers, 1972, and papers referred therein). Laboratory experiment of the direct interaction between electrons and neutrinos is under way by Gurr et al. (1972). Up to the present, they gave only an upper limit to the cross section, which is 1.9 times that of the universal Fermi interaction.

It is still meaningful to consider a limiting case, where the neutrino loss is completely neglected. Recently, Nomoto (1974) computed such a limiting case. Results for the star of $60 M_{\odot}$ with $\alpha_{p}=1.0$ are shown in Figure 4. Since the timescale of evolution is long enough for mixing, the mass of the silicon core is reduced by mixing below the Chandrasekhar limit, as anticipated by Stothers and Chin (1969). For a smaller mass star, the invasion of the convective envelope is somewhat weaker, because the effect of superadiabaticity is large for the smaller mass star (Nomoto and Sugimoto, 1972). The core of $20 M_{\odot}$ star evolves into the iron core, before its mass is reduced below the Chandrasekhar limit. For the star of $12 M_{\odot}$, the core mass is not reduced below the Chandrasekhar limit, until the photo-disintegration of iron takes place and the core becomes unstable. The above results are based on the assumption of $\alpha_{p}=1.0$. When $\alpha_{p}=1.5$ is assumed, the iron core of $12 M_{\odot}$ star is reduced below the Chandrasekhar limit by mixing.

In the core of mass smaller than the Chandrasekhar limit, electrons become degenerate. Convection in the envelope extends down to the active hydrogenburning shell, which surrounds the silicon or iron core. Hydrogen will be depleted uniformly in the envelope (Sugimoto, 1971). The lifetime of this phase of hydrogen depletion in envelope is estimated to be of the order of $10^{7} \mathrm{yr}$, which is longer than 


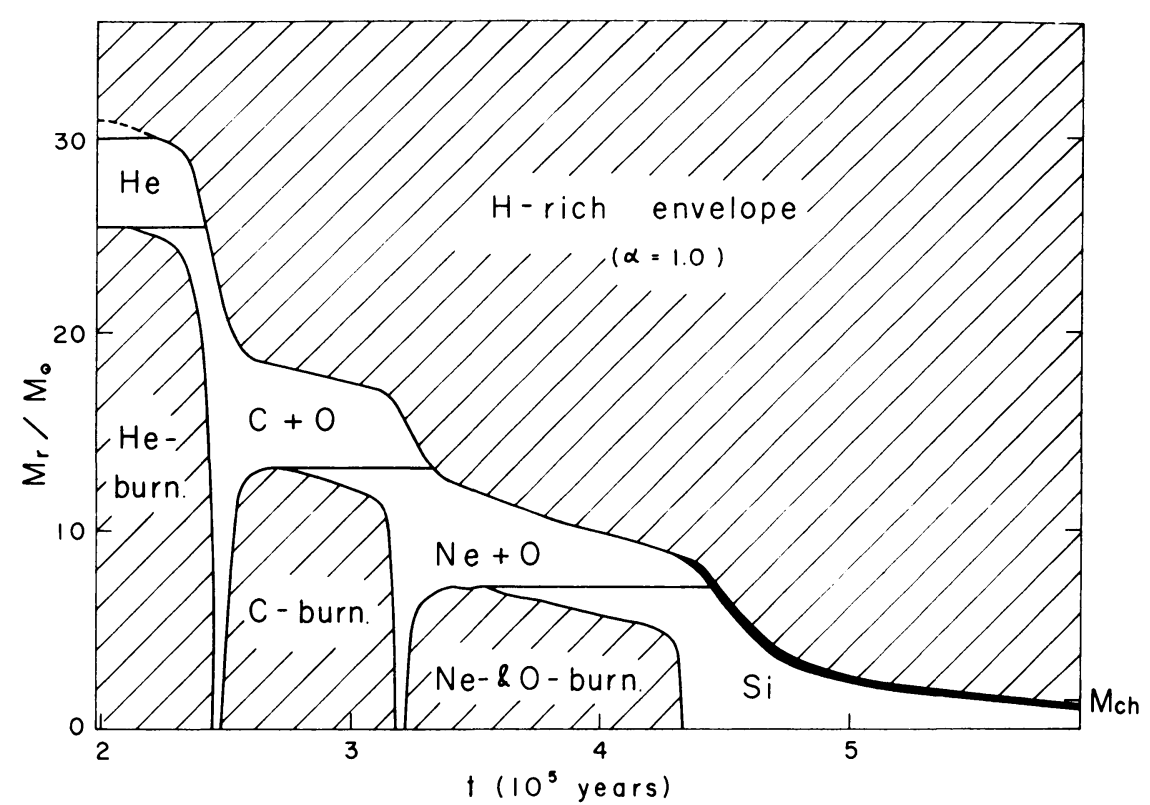

Fig. 4. The same as Figure 3, but for the star of $60 M_{\odot}$. Neutrino loss is neglected. Chandrasekhar's limiting mass is indicated by $M_{\mathrm{Ch}}$.

the lifetime of helium burning by a factor more than 20 . In this lifetime, the star has abundance peculiarity. Thus, if the neutrino loss were completely negligible, there would be as many peculiar stars as the massive main sequence stars (Stothers and Chin, 1969).

For the case of $12 M_{\odot}$ star with $\alpha_{p}=1.0$, the lifetime as a peculiar star is $5.7 \times 10^{4} \mathrm{yr}$, which extends from the penetration of convection into the carbon-oxygen zone up to the onset of dissociation of iron. The lifetime of this star from the contraction of carbon oxygen core up to the dissociation of iron is $7.2 \times 10^{5} \mathrm{yr}$. The lifetime as a red giant is somewhat longer than that, because the star spends a part of its heliumburning lifetime as a red giant. Then, the number of peculiar stars is somewhat less than $10 \%$ of normal red supergiants. In view of uncertainties involved, such a proportion of peculiar stars is not prohibitively high, because carbon stars and S-type stars consist $10^{-1}-10^{-2}$ of M-type giants (Iwanowska, 1966). We reach the following conclusion. The relative paucity of peculiar red supergiants is an evidence of the existence of the neutrino loss as discussed by Stothers and Chin (1969) and by Stothers (1972), unless the value of $\alpha_{p}$ is appreciably smaller than 1.0.

If we want to speak more strictly, the following comment should be amended. In all models computed above, the core mass is reduced below the Chandrasekhar limit after the oxygen-burning phase. Thus, the number of peculiar star is negligible, as long as the neutrino loss rate is larger than about $10^{-2}$ of the rate given by the universal Fermi interaction. 


\section{Stars of Intermediate Mass}

In this section we shall discuss the stars of masses in the range of 3-8 $M_{\odot}$, which evolve into the carbon detonation supernovae (Arnett, 1969; Paczyński, 1970a; Bruenn, 1971). These stars have a carbon-oxygen core in which electrons are strongly degenerate due to the neutrino cooling.

\subsection{EVOLUTION TOWARD THE CARBON DETONATION SUPERNOVAE}

When the central density becomes more than $10^{6} \mathrm{~g} \mathrm{~cm}^{-3}$, the convection becomes deeper and deeper. In the star of $5 M_{\odot}$, a part of helium in the intermediate zone is mixed into the outer envelope (Weigert, 1966). For more massive stars the convective envelope penetrates into the helium zone. The core mass is reduced to about $1.0 M_{\odot}$, if the mass of the helium core was larger than that value (Paczyński, 1970a; Sugimoto, 1971). In this phase of mixing, the hydrogen-burning shell remains inactive and the effect of superadiabaticity is small. Thus, Sugimoto's result is quantitatively consistent with Paczyński's.

As a result of the penetration of convection and the progress of the helium-burning shell, the hydrogen-burning shell comes very close to the hot helium-burning shell. Then, the hydrogen shell is ignited again. After this, the luminosity of the star increases and the effect of superadiabaticity becomes essential. Assuming $\alpha_{p}=1.0$, Paczyński (1970a, b) computed evolutionary sequence, where the convective envelope did not penetrate into the core beyond the active hydrogen-burning shell. As a result of simultaneous hydrogen and helium burning, the core mass grows close to the Chandrasekhar limit and explosive carbon burning takes place.

Assuming adiabatic convection, Sugimoto (1971) obtained a different picture of evolution, where the convective envelope penetrates into the core beyond the active hydrogen-burning shell.

Uus obtained some models by fitting detailed envelopes to isothermal cores. The convective envelope does not penetrate into the core for the case of $\alpha_{p}=1.0$ (Uus, 1970a), while the penetration is marginal for the star of mass $5 M_{\odot}$ with $\alpha_{p}=1.5$ (Uus, 1972c).

Since the penetration depends both on the assumption of $\alpha_{p}$ and on the stellar mass, it is time consuming to compute many cases for these parameters. Instead of this, Sugimoto and Nomoto (1973) computed growth of the core assuming the boundary condition similar to Equation (5). Such a boundary condition is found to be a good approximation as far as the bottom of convection is very close to the hydrogenburning shell in its temperature. Assuming no penetration of convection, they obtained the value of entropy at the core edge $\sigma_{1 e}^{(\text {cote })}\left(M_{1}\right)$ and the stellar luminosity $L\left(M_{1}\right)$ as functions of the core mass. As shown in Figures $5 \mathrm{a}$ and $5 \mathrm{~b}$, our $L\left(M_{1}\right)$ is close to Uus' result for $\alpha_{p}=1.5$, but somewhat different from Paczyński's and Uus' results for $\alpha_{p}=1.0$. The reason lies in the fact that the radiative zone is rather wide for the case of $\alpha_{p}=1.0$, which makes our boundary condition inappropriate.

When the hydrogen-burning stell is active, the temperature thereof is almost 
constant. Then the condition (3) against the penetration of convection is conveniently rewritten as

$$
L \lesssim L_{\text {mix }}\left(\alpha_{p}, M, M_{1}, T_{1}\right)<L_{\mathrm{cr}}\left(M_{1}\right) .
$$

Though some rough extrapolations were made, values of $L_{\text {mix }}$ were obtained using the work by Nomoto and Sugimoto (1972), which are shown in Figures 5a and 5b for different values of $\alpha_{p}$ and $M$. Inspection of these figures leads to the following conclusion: The penetration of convection does not take place in the case of

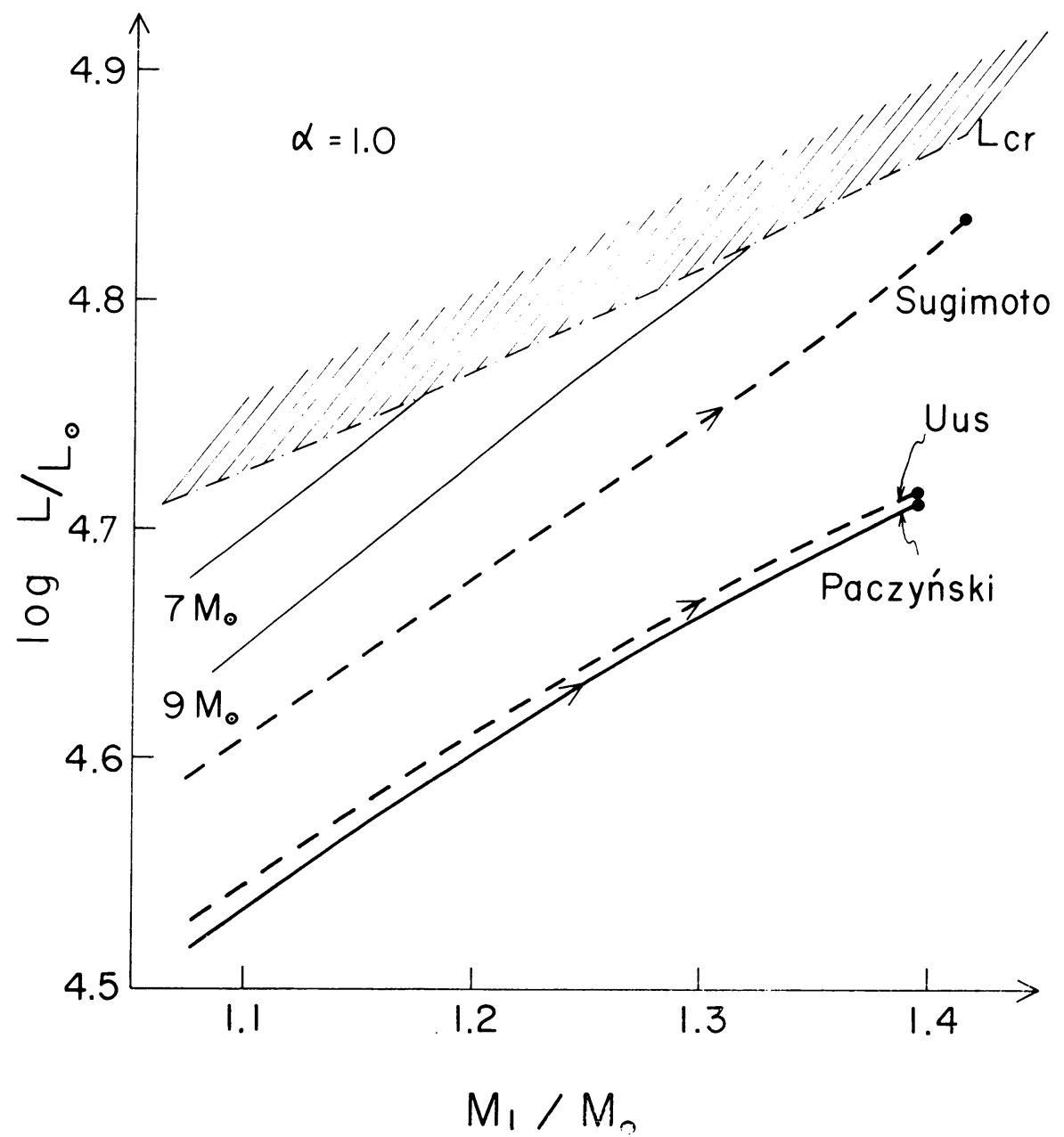

Fig. 5a. Evolutionary changes of stellar luminosity $L\left(M_{1}\right)$ (thick lines), the limiting luminosities against the mixing $L_{\text {mix }}\left(M_{1}, M\right)$ (thin lines labeled with stellar masses), local critical luminosity $L_{\mathrm{cr}}-$ $\left(M_{1}\right)$ at the core edge (dash-dot) are plotted against the mass of the growing electron-degenerate carbon-oxygen core. The case of $x_{p}=1.0$. Sugimoto's $L\left(M_{1}\right)$ is also included for comparison. No static solution is permitted in the shaded region. Convective envelope does not penetrate into the core when $L \leqslant L_{\operatorname{mix}}$. 


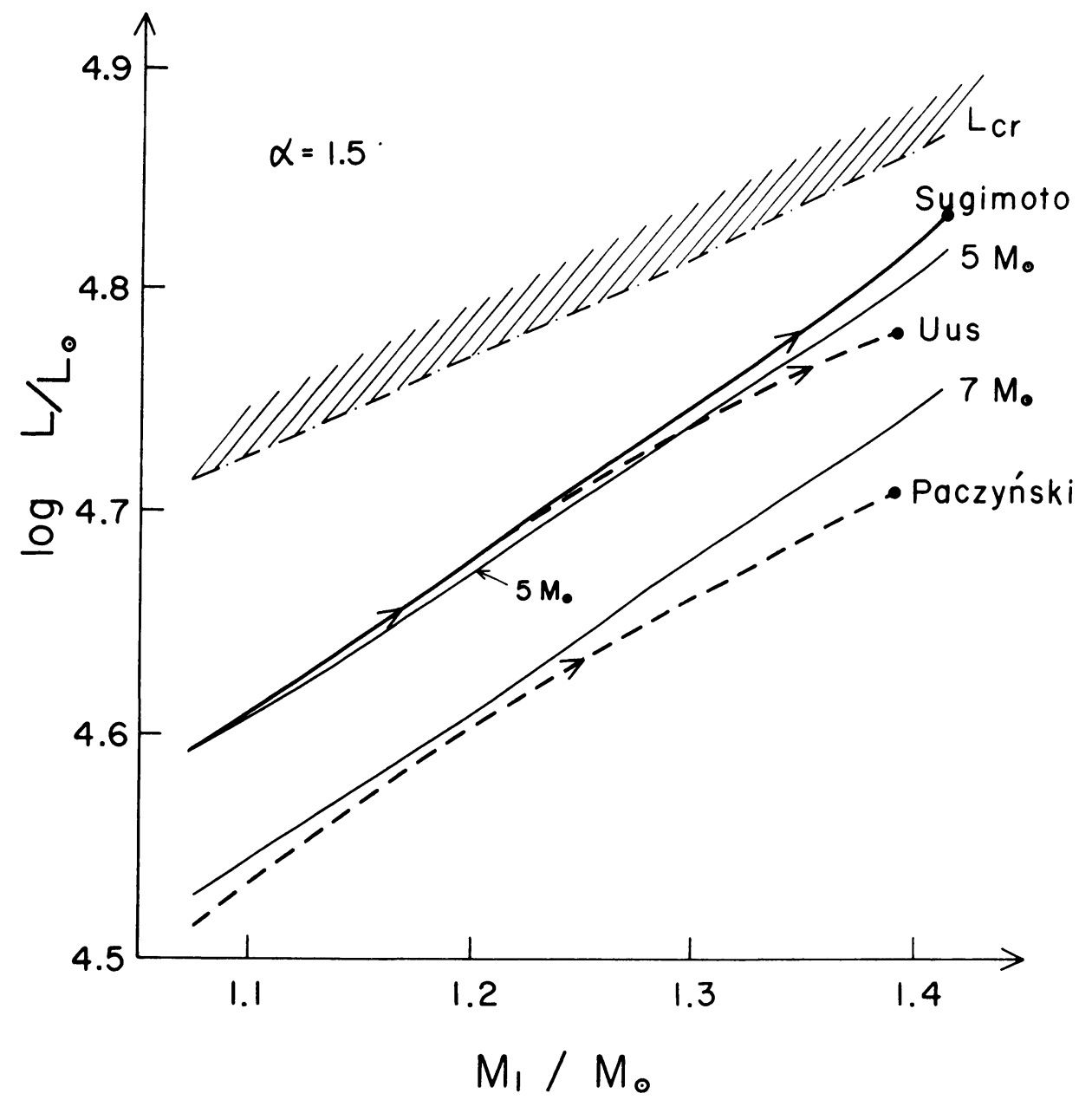

Fig. 5b. The same as Figure 5a, but for $x_{p}=1.5$.Paczyński's $L\left(M_{1}\right)$ is added for comparison.

$\alpha_{p}=1.0$ (Figure 5a), but it does take place for the star of mass greater than $5 M_{\odot}$ in the case of $\alpha_{p}=1.5$ (Figure 5b).

When the convection is penetrating into the core, material processed by the hydrogen-shell burning will be distributed throughout the envelope, and the hydrogen in the envelope is depleted uniformly. The lifetime of this phase of hydrogen depletion in envelope is $7 \times 10^{6} \mathrm{yr}$, up to the formation of the star having helium envelope (Sugimoto, 1971). Of this lifetime, the star spends about $\tau_{p} \simeq 5 \times 10^{6} \mathrm{yr}$ as a peculiar star, which has overabundance of carbon and oxygen. We shall assume that such a process happens for stars in the mass range of $\Delta m$ near $8 M_{\odot}$, where $m$ denotes stellar mass in units of $M_{\odot}$.

Stars of smaller mass evolve into the carbon detonation supernovae without the mixing. Using computations by Meyer-Hofmeister (1969), their lifetimes as red giants 
are approximately expressed by

$$
\tau_{r}(m)=2.2 \times 10^{7}(\mathrm{~m} / 5)^{-3.6} \mathrm{yr} .
$$

Taking into account the mass function proportional to $m^{-2.3}$ (Reddish, 1966), the ratio of the peculiar stars to normal red giants is expressed as

$$
8^{-2.3} \tau_{p} \Delta m / \int_{3}^{8} \tau_{r}(m) m^{-2.3} \mathrm{~d} m=6 \times 10^{-3} \Delta m .
$$

If we assume $\Delta m$ to be of the order of unity, the number of peculiar stars will be of the order of one percent, which is consistent with star counts by Iwanowska (1966).

\subsection{EFFECT OF THERMAL PULSES IN THE HELIUM-BURNING SHELL}

In all computations discussed above, the thermal instability of the helium-burning shell was artificially suppressed by assuming appropriate ratio of the energy generations by the helium- and the hydrogen-shell burnings. However, Uus (1970b) showed that the helium-burning shell is probably unstable.

Taking two models with $M_{1}=1.07$ and $1.39 M_{\odot}$ from the computation discussed in the preceding subsection, Sugimoto and Nomoto (1974) computed the development of thermal pulses. The case of $M_{1}=1.39 M_{\odot}$ is shown in Figure 6, which is qualitatively similar to those for the stars of $5 M_{\odot}$ (Weigert 1966) and of $1.0 M_{\odot}$ (Sweigart, 1971), both of which have the core of $M_{1} \simeq 0.8 M_{\odot}$. Convection appears in the helium zone and the helium is redistributed as shown in Figure 7. After the peak of the helium burning, the helium zone expands and the hydrogen-burning shell is extinguished. As shown in Figure 6, entropy at the edge of the core increases to a very large value, until the condition against mixing (3) is violated.

Though the progress of mixing has not been computed, it will develop as follows. As the mixing proceeds, the hydrogen-burning shell will move inward in mass. Then the temperature will rise and the hydrogen-shell burning will be ignited again. At the same time, the entropy at the core edge will decrease and the penetration of convection will stop.

As seen in Figure 7 the products of helium burning are distributed almost all over the helium zone. The width of the zone consisting of pure helium is relatively smaller for the pulses with a larger core mass. When the core mass is larger than a certain value, the products of helium burning may be brought to the stellar surface by the penetrating convection. This implies that the stars with luminosity higher than a certain value will be peculiar. Unfortunately, the proportion of the peculiar stars cannot be predicted, until detailed models with penetrating convection are computed.

\section{Small Mass Stars}

The stars of mass around $1 M_{\odot}$ have a phase of electron-degenerate helium core. When such a star reaches the red giant branch of globular clusters, the surface 


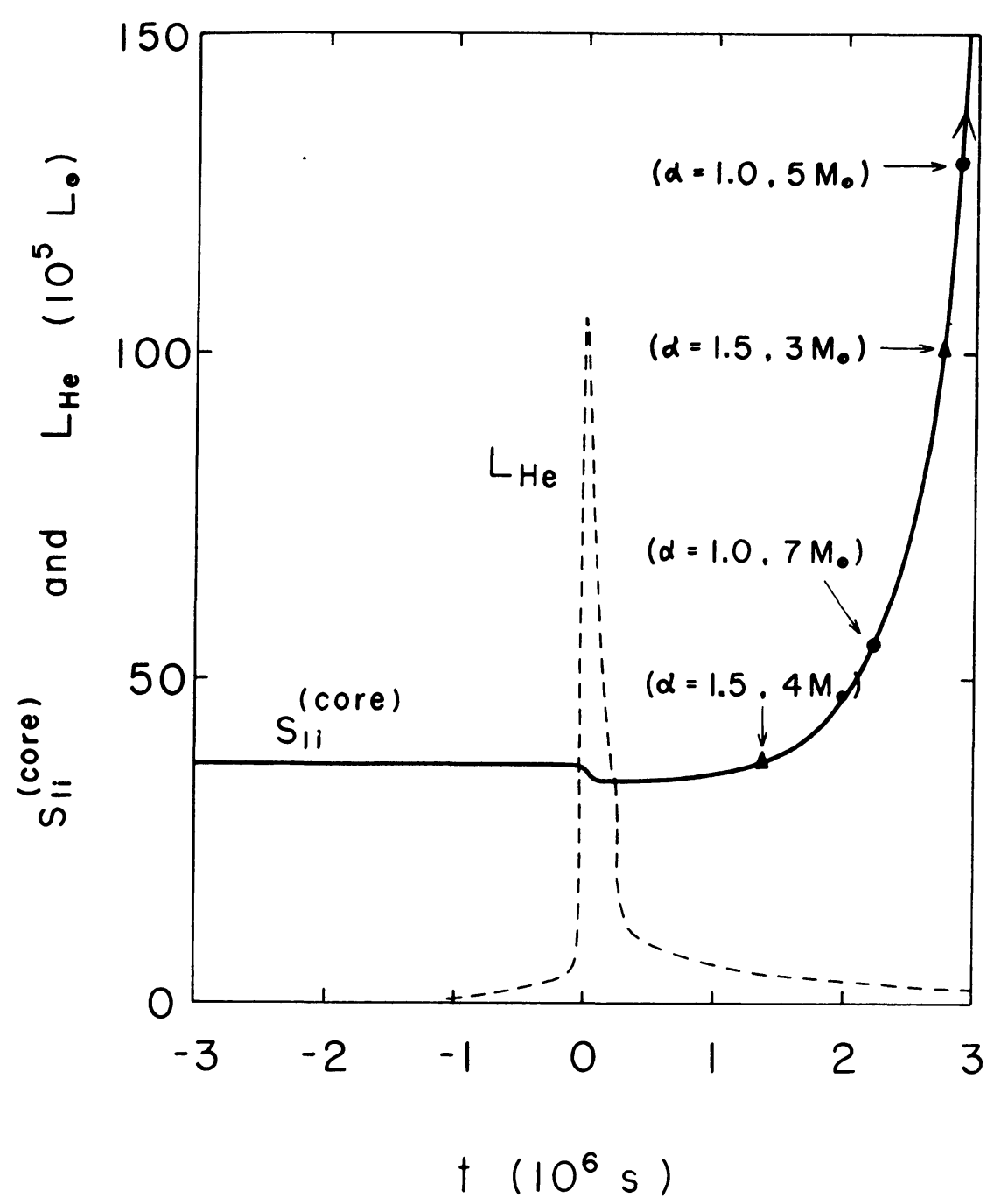

Fig. 6. Thermal pulse of the helium-burning shell for the carbon-oxygen core of mass $1.39 M_{\odot}$. Change of entropy at the core edge and the energy-generation rate of the helium burning $L_{\mathrm{He}}$ are shown. Critical values of entropy are shown by dots and triangles for different cases. For the entropy above the critical value, the convective envelope should have penetrated into the core.

convection zone becomes deeper. The convection penetrates into the core and the helium content of the envelope is increased by about $2 \%$ (Thomas, 1967; Demarque and Mengel, 1971). After the thin hydrogen-burning shell is formed, the convection does not penetrate any more.

Just after the helium flash, Thomas found a partial mixing between the core and envelope, but Demarque and Mengel did not. This difference came from the fact that Thomas used the neutrino loss rate too large by a factor of 4 , and that the helium flash took place in a relatively outer shell. 


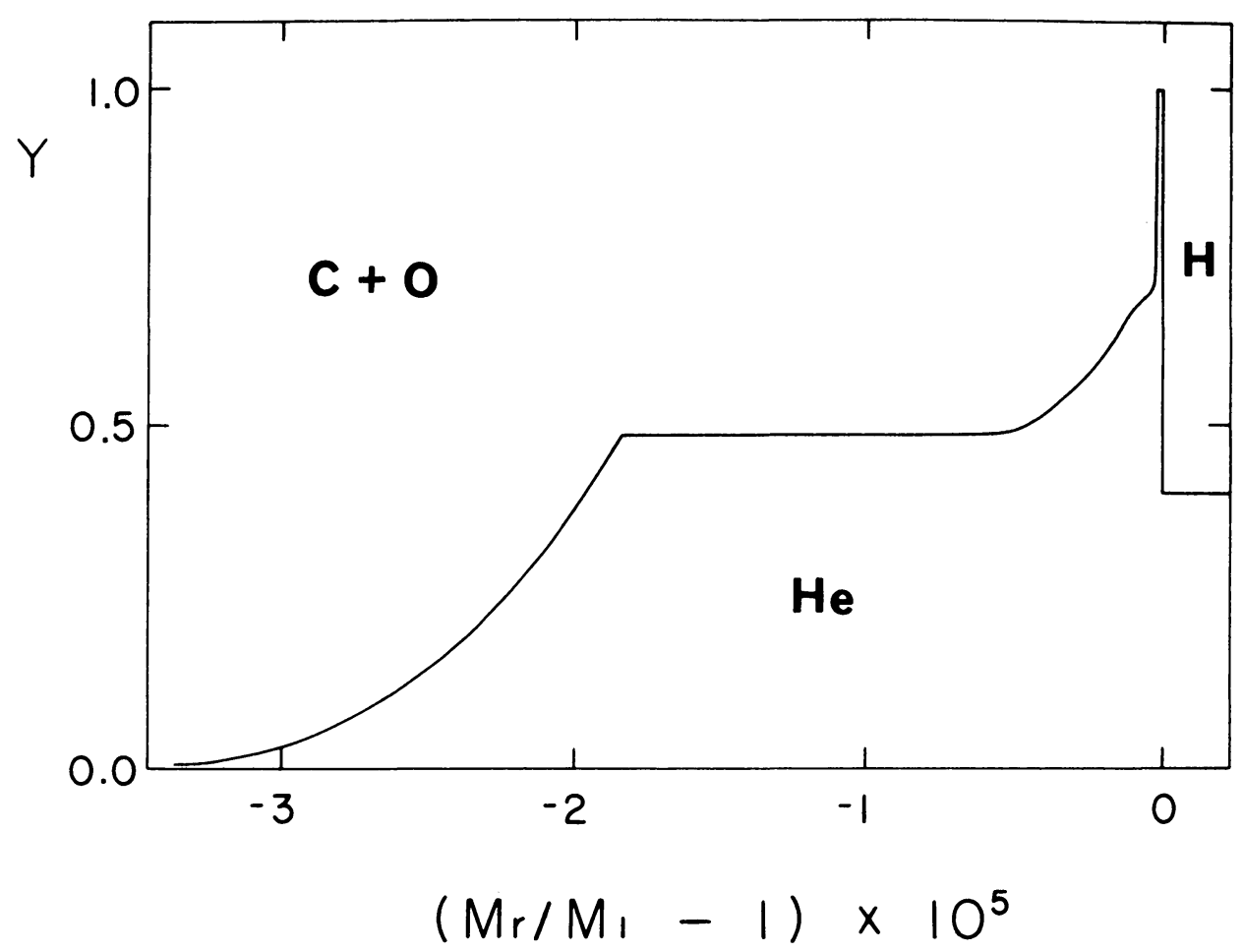

Fig. 7. Distribution of helium concentration $Y$ in the thin helium zone of the star for a stage after the peak of the thermal pulse.

After the core helium burning, the thermal instability in the helium-shell burning takes place as discussed in Section 5.2. Schwarzschild and Härm (1967) found that the convection appearing in the pulsing helium shell touches the tail of the hydrogen distribution. In this case the hydrogen is brought into the helium zone, but nothing is brought into the surface convection zone, because the surface convection zone is too shallow. Sweigart (1971) obtained a similar result for a later phase of the same star. Unfortunately, their results cannot be compared with ours in Section 5.2, because they did not include the effect of radiation pressure and entropy of radiation, which play important roles for the problem of mixing especially in later phases of evolution.

For the star of mass between 4 and $1 M_{\odot}$, the total energy of the red giants' envelope becomes positive and the envelope becomes pulsationally unstable, if the luminosity of the red giant exceeds a certain critical value $L_{0}$. Paczyński and Ziolkowski (1968) proposed this to be a cause of Mira type variables, mass loss from the red giants and formation of planetary nebulae. Such an effect takes place, if the mass of the star is smaller than a critical mass $M_{0}$. Assuming $\alpha_{p}=1.0$, they determined approximate value of $M_{0}$ to be $4 M_{\odot}$.

We have repeated such computation as done by them, using different values of $\alpha_{p}$. For the range of $\alpha_{p}=1.5-0.7, M_{0}$ lies in the range of 3-4 $M_{\odot}$, though a somewhat 
larger $L_{0}$ corresponds to a larger $\alpha_{p}$ (Fujimoto et al., 1973). The critical mass is rather insensitive to $\alpha_{p}$, because almost all mass of the envelope is in the ionization zone irrespective of the value of $\alpha_{p}$.

Apart from the mass loss, the penetration of convection into the core is less likely, because the effect of superadiabaticity is larger for the stars of smaller mass (Nomoto and Sugimoto, 1972). When mass loss takes place, the core mass and the stellar luminosity hardly change, while the stellar mass becomes smaller. Then, the convection is more difficult to penetrate into the core.

\section{Concluding Remarks}

Though the general properties of the deepening convective envelope were discussed, uncertainty is left in the parameter of the mixing length theory. Moreover, quantitative results depend also on details of stellar models, especially on the distribution of hydrogen both in the intermediate zone and within the thin hydrogen-burning shell. Mechanism of penetration of convection beyond the barrier of the change in the mean molecular weight awaits for detailed investigation.

However, the effect of the parameter $\alpha_{p}$ is relatively small, when the stellar luminosity is relatively small compared with the critical luminosity at the bottom of the envelope, and when the temperature at the bottom of the envelope is relatively low and the hydrogen-shell burning is inactive. In such cases and in some extreme cases, we can draw conclusions with some confidence.

For the star of mass $60 M_{\odot}$, the convection penetrates into the carbon-oxygen zone and the star becomes a peculiar star. For the star of mass in the range of 8-30 $M_{\odot}$, the convection penetrates, at the deepest, only to the helium zone. However, there exist carbon and S-type stars more luminous than $1 \times 10^{5} L_{\odot}$ (Gordon, 1968; Motteran, 1971). They seem to exceed the maximum luminosity that can be attained by the star evolving toward the carbon detonation supernova. These carbon and S-type stars should be attributed to stars of mass larger than $8 M_{\odot}$ or stars devoid of hydrogen. We must seek other mechanisms to interpret these highly luminous peculiar stars. If the neutrino loss were completely negligible, on the other hand, there would be too many peculiar stars, as far as the $\alpha_{p}$ is not much smaller than 1.0.

For the star of mass in the range of 8-3 $M_{\odot}$, there are two possibilities of the origin of peculiar stars. One is the hydrogen depletion in the envelope. This type of origin is interesting, because it may be connected with the origin of the star having helium envelope and the hydrogen deficient carbon stars such as $\mathrm{R} \mathrm{CrB}$. The other possibility is the mixing which takes place just after each cycle of the thermal pulses (see also Scalo and Ulrich, 1973). However, the number of peculiar stars cannot be predicted at present, for the above two possibilities.

There exist carbon stars of luminosities fainter than $10^{4} L_{\odot}$ (Gordon, 1968). Such stars seem to be attributed to the stars of relatively small mass. Effect of thermal pulses for such small mass stars are still an open question. 


\section{References}

Arnett, W. D.: 1969, Astrophys. Space Sci. 5, 180.

Böhm-Vitense, E.: 1958, Z. Astrophys. 46, 108.

Bruenn, S. W.: 1971, Astrophys. J. 168, 203.

Chandrasekhar, S.: 1939, An Introduction to the Study of Stellar Structure, The University of Chicago Press, Chicago; 1957, Dover Publ., New York.

Demarque, P. and Mengel, J. G.: 1971, Astrophys. J. 164, 317.

Eggleton, P. P.: 1967, Monthly Notices Roy. Astron. Soc. 135, 243.

Fujimoto, M., Nomoto, K., and Sugimoto, D.: 1973, private communication.

Gordon, C. P.: 1968, Publ. Astron. Soc. Pacific 80, 597.

Gurr, H. S., Reines, F., and Sobel, W.: 1972, Phys. Rev. Letters 28, 1406.

Hayashi, C. and Hōshi, R.: 1961, Publ. Astron. Soc. Japan 13, 442.

Hayashi, C., Hōshi, R., and Sugimoto, D.: 1962, Prog. Theor. Phys. Kyoto Suppl., No. 22, 1.

Hoyle, F. and Schwarzschild, M.: 1955, Astrophys. J. Suppl. $2,1$.

Iwanowska, W.: 1966, in M. Hack (ed.), Colloquium on Late-Type Stars, p. 398.

Meyer-Hofmeister, E.: 1969. Astron. Astrophys. 2, 143.

Motteran, M.: 1971, in M. Hack (ed.), Colloquium on Supergiant Stars, p. 292.

Nomoto, K.: 1974, to be published in Prog. Theor. Phys. Kyoto 52.

Nomoto, K. and Sugimoto, D.: 1972, Prog. Theor. Phys. Kyoto 48, 46.

Paczyński, B. and Ziolkowski, J.: 1968, Acta Astron. 18, 255.

Paczyński, B.: 1969, Acta Astron. 19, 1.

Paczyński, B.: 1970a, Acta Astron. 20, 47.

Paczyński, B.: 1970b, Acta Astron. 20, 287.

Reddish, V. C.: 1966, Vistas in Astronomy 7, 173.

Scalo, J. M. and Ulrich, R. K.: 1973, Astrophys. J, 183, 151.

Schwarzschild, M. and Härm, R.: Astrophys. J. 150, 961.

Stothers, R. and Chin, C.-W.: 1969, Astrophys. J. 158, 1039.

Stothers, R.: 1972, Astrophys. J. 175, 717.

Sugimoto, D.: 1970a, Prog. Theor. Phys. Kyoto 44, 375.

Sugimoto, D.: 1970b, Prog. Theor. Phys. Kyoto 44, 599.

Sugimoto, D.: 1971, Prog. Theor. Phys. Kyoto 45, 761.

Sugimoto, D. and Nomoto, K.: 1974, in preparation.

Sweigart, A. V.: 1971, Astrophys. J. 168, 79.

Thomas, H. C.: 1967, Z. Astrophys. 67, 420.

Uus, U.: 1970a, Nauch. Inform. Akad. Nauk SSSR 17, 3.

Uus, U.: 1970b, Nauch. Inform. Akad. Nauk SSSR 17, 48.

Uus, U.: 1971, Nauch. Inform. Akad. Nauk SSSR 20, 64.

Uus, U.: 1972a, Nauch. Inform. Akad. Nauk SSSR 21, 46.

Uus, U.: 1972b, Nauch. Inform. Akad. Nauk SSSR 21, 51.

Uus, U.: 1972c, Nauch. Inform. Acad. Nauk SSSR 23, 85.

Weigert, A.: 1966, Z. Astrophys. 64, 395.

Ziolkowski, J.: 1972, Acta Astron. 22, 327. 